\title{
TEORÍA DE LOS LUGARES CENTRALES EN MALLORCA REVISITADA
}

\author{
Margalida Mestre Morey \\ Departamento de Geografía \\ Universidad Autónoma de Barcelona \\ margarita.mestre@e-campus.uab.cat
}

\section{RESUMEN}

El articulo analiza el sistema urbano de Mallorca a partir de los establecimientos de venta al por menor y los servicios entre 1970 y 2011. Para ello, se toma como referencia la metodología de Brian Berry (1971) y su aplicación pionera en Mallorca por Alberto Quintana (1979). Así, ha podido llegarse a la conclusión que 40 años más tarde la estructura del sistema urbano se ha modificado profundamente, debido, sobre todo, a la mayor dispersión de población y establecimientos, lo cual ha propiciado una redistribución de la jerarquía de municipios, altamente influenciada por el turismo.

Palabras clave: establecimientos al por menor, Mallorca, Albert Quintana, teoría de los lugares centrales, Brian Berry.

\section{ABSTRACT}

The article analyzes the urban system of Mallorca from the local retail and services between 1970 and 2011. For it, is taken as reference Brian Berry's methodology (1971) and its pioneering application in Mallorca by Alberto Quintana (1979). From this analysis concludes that, 40 years later, the structure of the urban system has changed radically, due mainly to the greater dispersion of population and facilities, which has led to a redistribution of the hierarchy towns, highly influenced by tourism.

Keywords: retail establishments, Mallorca, Albert Quintana, central place theory, Brian Berry.

Fecha de recepción: julio 2014.

Fecha de aceptación: marzo 2015. 


\section{INTRODUCCIÓN}

Para comprender la evolución de un territorio es necesario, obviamente, considerar el máximo de dinámicas que lo rigen, una de ellas es el comercio al por menor y del sector servicios. Como dijo Brian Berry:

«Las empresas dedicadas al comercio minorista y las de servicios son el último eslabón de la cadena de producción y distribución y el primer paso en el proceso del consumo. En la geografía de las empresas comerciales minoristas y de las empresas de servicios es donde radica la movible línea divisoria, el dinámico diafragma que separa la geografía de la producción, que ha sido por mucho tiempo un tema de interés profesional y de estudio, y la hasta ahora poco cultivada geografía del consumo» (Berry, 1971: 2).

Como es sabido, Berry creó un método a partir de la teoría de los lugares centrales de Christaller y Lösch ${ }^{1}$, con el objetivo de explicar la localización del comercio al por menor y los servicios considerando que «la distribución geográfica de los comercios minoristas y de las empresas de servicios en los lugares centrales se ajusta aproximadamente a la distribución geográfica de los consumidores» (Berry, 1971: 7). Así, el volumen de la población que reside en cada municipio tendría una relación directa con el número y la diversidad de comercio al por menor y los servicios que en ellos se hallan.

Berry desarrolló sus estudios por áreas típicamente representativas de la parte central de Estados Unidos² (Berry, 1971: 4). En el Estado Español una aplicación pionera de esta metodología fue la tesis doctoral del historiador, reconvertido a geógrafo, Alberto Quintana, El Sistema Urbano de Mallorca (1979), donde aplicó el método de Brian Berry por primera vez en Mallorca con datos de 1970. La tesis de Quintana publicada en el 1979, por la ya desaparecida editorial Moll, ha sido considerada un hito en los estudios de geografía urbana y en la evolución del conjunto de la disciplina en nuestro país. Así, en 2003, Horacio Capel afirmaba que «dicha tesis constituye una obra importante por su método, innovador en el contexto de la geografía española, y por su crítica epistemológica de la geografía dentro de la ciencia en general»(Capel, 2003: 30).

1 Sobre la obra de Christaller, véase Die zentralen Orte in Süddeutschland (1933) en la que explica y aplica, para la Alemania meridional, la Teoría de los Lugares Centrales. En cuanto a su aplicación en España, uno de los primeros análisis de centralidad fue el de Estébanez (1974), «Cuenca. Estudio geográfico», el cual está conectado con otra tesis posterior de López (1973), Lugares centrales y zonas de influencia en el Noroeste de España. Seguidamente tiene lugar el trabajo pionero de Quintana (1979) para la isla de Mallorca y el de Pilar Riera (1988) Les àrees funcionals de Catalunya. Otra aplicación de un método que mide la centralidad, estrechamente relacionado con la Teoría de los Lugares Centrales, es el método Rank-size, aplicado para la isla de Mallorca por Rullan (1979) el cual lo utiliza para conocer la distribución de la previsión de suelo en Mallorca. Por otra parte, para conocer la obra de Lösch (1944), véase la «Teoría Económica Espacial» a partir de la cual introduce una serie de variantes a la Teoría de los Lugares Centrales, como por ejemplo la posibilidad de especializaciones productivas de los centros.

2 «El área 1 abarca el área metropolitana de Chicago, incluyendo la ciudad y los suburbios. El área 2 es un típico ejemplo de las comarcas ganaderas y de cultivo de maíz del Midwest, y se extiende entre Omaha-Council Bluffs al Oeste y Des Moines al Este, en la parte sudoccidental de Iowa. Las áreas 3 y 4 se encuentran en Dakota del sur (...). Cada una de estas áreas fue analizada con detalle (realizando extensos trabajos sobre el terreno) en 1960 , en un estudio encaminado a valorar las propiedades de los sistemas de «lugares centrales» en una gran variedad de distintos emplazamientos, y comparar sus propiedades en las diversas áreas» (Berry, 1971: 4-5) 
El objetivo principal de este artículo consiste en la recuperación de la obra de Quintana para contrastarla con los datos de 2011 y poder observar la evolución de la cuestión por él estudiada en los últimos 40 años. Asimismo, el trabajo quiere ser una contribución a la utilización crítica de los métodos de Brian Berry respecto la geografía del comercio y del consumo, y también al análisis del sistema comercial de la isla iniciado en el año 1963 con el Altas Comercial de España (Fontana, 1963) y seguido por la memoria presentada por la Cámara de Comercio en el año 1964 (COCIN, 1964), en la cual aparecía el primer estudio local sobre las áreas de influencia comercial (Rullan, 2002).

Para ello se pretende, en primer lugar, explicar de forma consecuente el método de Brian Berry, a partir de su libro Geografía de los centros de mercado y distribución al por menor (1971). A continuación, analizar la aplicación que Alberto Quintana hace de éste en su tesis doctoral y, vistos los antecedentes, nos adentraremos a mostrar y contrastar con los datos de 2011 la evolución de la distribución del comercio al por menor y los servicios. Además, en último lugar, se actualizarán para 2011 dos tipologías clasificatorias de los municipios a partir de los mismos datos, creadas por el autor mallorquín en 1970, donde se destaca el peso del turismo en Mallorca y la alteración acaecida en la jerarquía urbana.

La hipótesis que trataremos de demostrar es que, en los últimos 40 años, se ha producido una pérdida de centralidad que ha conllevado y conllevará en un futuro profundos cambios en las características que hasta ahora han definido el sistema urbano mallorquín, como es la imponente macrocefalia. Se estaría transitando así, desde un sistema caracterizado por una destacada centralidad, la cual tiene como componente principal la capital insular, a la concepción de integración del conjunto de la isla.

El artículo se divide en los siguientes apartados: la introducción, el método de Brian Berry, los antecedentes y la reivindicación de la obra de Alberto Quintana, el análisis comercial elaborado por el autor mallorquín, la actualización de los datos para 2011, las correlaciones, las tipologías del equipamiento y, finalmente, unas conclusiones.

\section{EL MÉTODO DE LOS ESTABLECIMIENTOS AL POR MENOR Y LOS SERVICIOS DE BRIAN BERRY}

Brian Berry enmarcó la teoría de los lugares centrales de Christaller y Lösch dentro de su obra La geografía de los centros de mercado y la distribución al por menor la cual fue publicada en el año 1941. La hipótesis de partida del autor es que «la esencia de la geografía de las empresas de servicios y el comercio al por menor estriba en el 'agrupamiento' de establecimientos en los centros de mercado frecuentados por los consumidores de la zona» (Berry, 1971: 3) debido a que, como dice el mismo autor «la cualidad esencial de un foco es su centralidad» (Berry, 1971: 4), por ello, la teoría de los lugares centrales es «la base teórica de una gran parte de la geografía urbana y de la geografía del comercio al por menor y de las empresas de servicios» (Berry, 1971: 4), mostrando así la tradición científica en la que la obra del autor británico pretende inscribirse.

Como es sabido, la teoría de los lugares centrales fue formulada por Walter Christaller en Alemania en 1933 y a la cual August Lösch introdujo nuevos conceptos en 1944. Pese a las posibles modificaciones la finalidad de la TLC es la de explicar, a partir de ciertos principios generales, la distribución y jerarquización de los espacios urbanos que dan determinados servicios a la población de un área circundante en un espacio isotrópico. 
A partir de estas premisas, Berry (1971) explica que en las sociedades económicamente avanzadas, las actividades de producción, distribución (intercambio) y consumo tienden a diferenciarse de manera nítida. El intercambio está enmarcado dentro del proceso de distribución entre productores y consumidores dentro del mercado, entendiendo el mercado como un sistema de producción de precios que se autorregula; los precios, al mismo tiempo, constituyen el mecanismo que conecta los actos individuales de elección.

El hecho de centrar su interés en las empresas dedicadas al comercio minorista y a los servicios, se explica, porque estas representan un último escalón de la cadena de distribución y producción y son el primer paso dentro del proceso de consumo. Por tanto, el comercio minorista y los servicios son «la fina línea divisoria» entre la geografía de la producción y la geografía del consumo (Berry, 1971: 2).

Para conocer si el comercio minorista y los servicios en Iowa se regían por criterios de centralidad, Berry realizó una serie de correlaciones (1971: 44-51) a partir de las variables: población, diversidad de establecimientos y número de establecimientos, dos de las cuales fueron utilizadas posteriormente por Quintana para la isla de Mallorca.

a. Correlación entre el número de establecimientos y la diversidad de establecimientos: cuantos más tipos diferentes de establecimientos tiene un municipio más diversidad existe.

b. Correlación entre número de establecimientos y población: tal como cita el mismo autor, siguiendo el concepto de centralidad que define la TLC, «el índice de crecimiento de los establecimientos es proporcional al índice de crecimiento de la población del centro» (Berry, 1971: 48).

De esta forma llega a la conclusión general que la distribución geográfica del comercio al por menor y de las empresas de servicios en los lugares centrales se ajusta aproximadamente a la distribución geográfica de los consumidores (Berry, 197: 7).

\section{LOS ANTECEDENTES EN EL ESTUDIO DE LA GEOGRAFÍA DE MALLORCA Y LA REIVINDI- CACIÓN DE ALBERTO QUINTANA}

Alberto Quintana fue un gran reivindicador de los estudios urbanos dentro de la geografía mallorquina ${ }^{3}$, y también contribuyó a la transformación del marco teórico de esta disciplina en España. Como el propio autor explica «tal transformación vino motivada por el deseo - acentuado en los geógrafos que accedieron a primer plano en torno a 1955-de desprenderse de los lastres que nuestra ciencia arrastraba como consecuencia de su peculiar origen.»(Quintana, 1974: 55).

El empeño de Quintana, que había estudiado historia en la Universidad de Salamanca, y se terminó doctorando en geografía en el año 1975, siendo discípulo de Bartomeu Barceló,

3 Sobre la trayectoria y obra de Alberto Quintana, autor clave en la evolución de los estudios de geografía urbana de las Islas Baleares, véase el conjunto de trabajos reunidos en Albert Quintana: el seu temps i la seva obra (Artigues, et al, 2008). La obra cuenta con aportaciones de Pere Antoni Salvà Tomas, Emilio Alonso, Horacio Capel, Antonio J. Campesino, Florencio Zoido i Oriol Nel·lo. 
era introducir un cambio en los estudios de geografía urbana, pasando de un método clásico a una nueva geografía que reivindicaba la formulación de hipótesis y teorías, y la elaboración de conceptos y leyes, para que fueran de aplicación general.

Los postulados de Quintana suponen un cambio destacado en la forma de abordar la geografía urbana, frente a la generación de estudiosos, que hasta el momento, la habían cultivado en la isla de Mallorca. Con la superación de las descripciones literarias de los grandes autores que explicaban el paisaje insular, geógrafos como Bisson (1964) y Deffontaines (1967) describían la fisionomía de la isla a partir de la agricultura incorporando, por primera vez, la población. Fue el propio director de tesis de Quintana, Bartomeu Barceló (1968), que introdujo el peso poblacional y turístico entre las variables para la creación de la primera variación del mapa comarcal de Vicenç M. Rosselló i Verger (1964). Rosselló fue autor de una de las primeras comarcalizaciones de la isla ${ }^{4}$, la cual, pese a tratarse de un análisis y clasificación de los municipios en función del regadío en Mallorca, resultó de enorme influencia sobre los análisis territoriales y sobre la organización de los municipios mallorquines (Rullan, 2002: 263). En los siguientes años Barceló (1970) trabajó ampliamente los datos de población en su tesis doctoral, iniciando una especialización temática que se consolidó con la tesis de Quintana (1979).

Así pues, es a partir de estos autores y muchos otros también vitales, aunque aquí no nombrados -como Ferrer (1974), Salvà (1978), Florit (1983), entre otros-, que se consolida el nuevo paradigma reclamado por Quintana. De esta forma, como dijo Capel (2008: 44) el trabajo de Quintana «era ambicioso no sólo por la aportación específica sobre Mallorca, sino por su voluntad de contribuir a la renovación de la geografía española».

\section{ALBERTO QUINTANA Y EL ANÁLISIS DEL EQUIPAMIENTO COMERCIAL EN 1970}

Como ya sabemos, los estudios de Quintana tuvieron por finalidad utilizar, para la isla de Mallorca, una serie de técnicas cuantitativas y procedimientos estadísticos que hasta el momento habían sido poco utilizados en el conjunto de España y nunca para la mayor de las islas Baleares.

En su tesis «El Sistema Urbano de Mallorca» aplicó, entre otras técnicas, el método de Berry (1971) al que nos hemos referido, ya que según el autor mallorquín «la teoría de los lugares centrales o las correlaciones de Berry, ha tenido un valor innegable, abriendo fecundas vías de investigación, intentando comparaciones y regularidades y contribuyendo a un mejor conocimiento de la forma en que determinados fenómenos se distribuyen en espacios concretos» (Quintana, 1979: 17-18).

Así en el apartado referido al equipamiento comercial de su tesis doctoral, el autor analiza las empresas existentes en cada municipio dedicadas a la distribución de bienes o a la prestación de servicios al por menor usando, como base para su estudio, la Matricula Industrial de Mallorca de 1970. El autor trabajó, concretamente, con el número de establecimientos comerciales y también su clasificación en diferentes ramas (alimentación, textiles, etc.), lo que le permitió conocer la diversidad de establecimientos con los que contaba la isla.

4 Entre las primeras comarcalizaciones de la isla está la de Edmunt W. Gilbert (1934). 
En aquel momento, la Matricula Industrial de 1970 tenía contabilizados 32.268 establecimientos divididos en 18 grupos diferentes, considerados por el propio Quintana un esquema poco diversificado, y el censo de población de 1970 tenía cuantificados 460.030 habitantes (Quintana, 1979: 87).

Es a partir de estos datos que el autor aplica para la isla dos de las correlaciones de Berry, contrastando, por una parte, la diversidad de establecimientos y su cantidad y, por otra parte, la cantidad de establecimientos y la población.

Quintana define el primer resultado $(\mathrm{r}=0,737)^{5}$ como una «relativamente baja correlación entre la diversidad y número de establecimientos», el cual apunta hacia una elevada especialización en determinados grupos de establecimientos, consecuencia de la escasa diversificación. Así pues, que un municipio tenga un elevado número de establecimientos no tiene porqué repercutir en una mayor diversidad, como afirmaba Berry. Además, contrastando sus resultados con los del autor Británico, Quintana afirma que debido a la imposibilidad de llegar a una jerarquización de municipios que responda a cuantos más establecimientos, más tipos de establecimientos existen, nos encontrábamos «ante un área fuertemente urbanizada» (1979: 89).

En cuanto a la segunda correlación, entre el número de establecimientos y la población, el resultado es perfecto $(r=0,997)$. Así, es el propio Quintana quien cita a Berry (1971) remarcando que «el número total de establecimientos comerciales y de servicios radicados en un centro es en función de la población del mismo», pero el autor también describe su asombro, «ya que parecía deducirse de lo dicho en la observación anterior que estábamos ante un caso de especialización» (Quintana, 1979: 90). Esta perfecta relación entre las dos variables, rompe ya una lanza a favor de la integración del sistema urbano, debido a que no existen aparentes desequilibrios que muestren que gran parte de la población de la isla necesite un núcleo de mayor rango comercial. Parece indicar que la macrocefalia ejercida por Palma no desempeña una influencia tal sobre el resto del sistema urbano que desvirtúe su cantidad de establecimientos. Lo cual lleva al autor a preguntarse si la capital regional se encuentra fuera de la isla (Quintana, 1979: 90).

Para profundizar más en el análisis, el autor mallorquín realizó una segunda correlación dividida entre municipios turísticos $(r=0,725)$ y no turísticos $(r=0,951)$, lo cual le permitió comprobar que «los centros turísticos no constituyen, "grosso modo», lugares centrales porque la población en ellos reunida no se corresponde con la cantidad de establecimientos comerciales que concentran» (1979: 90), mientras que en los municipios no turísticos la correlación es perfecta, de la misma forma que en el conjunto de la isla, mostrándose así una importancia indirecta del turismo en la isla.

5 Rehaciendo los cálculos a partir de los datos de 1970 con los que trabajó Quintana, se observa una disonancia entre el coeficiente de correlación que aparece en la publicación de la tesis $(r=0,737)$ y el que se obtiene rehaciendo de nuevo los cálculos $(\mathrm{r}=0,383)$. Esta discordancia puede deberse a dos razones: en primer lugar, aquella que parece más probable, que se trate de una errata tipográfica; en segundo lugar, que se trate de un error de cálculo. Esta última posibilidad nos parece poco atendible debido al rigor y minuciosidad con la que trabajaba el autor y, aún más, con el hecho de que él mismo afirma que se trata de una «relativa baja correlación» (Quintana, 1979: 89) cosa que no acabaría de concordar con un resultado de $\mathrm{r}=0,737$. No hemos conseguido encontrar los papeles originales después de haber indagado en la Universitat de les Illes Balears, Universitat de Barcelona y en el archivo personal de Bartomeu Barceló. 
Con la aplicación de esta metodología el autor concluyó que «la Teoría de los Lugares Centrales solo puede ser aplicada en áreas con una población rural más o menos regularmente distribuida y donde no existan funciones especializadas como la del turismo, que alteren la centralidad de los lugares»(Quintana, 1979:89). Con ello sus conclusiones contrastan con el estudio que Estébanez (1974) realizó para Cuenca, el cual constituye el primer análisis de centralidad para España (López, 1987) y a partir del que pudo mostrar que para esta provincia si existe una distribución del número de habitantes, de establecimientos y de grupos de establecimientos acorde con la definición de centralidad. Es muy significativo que Quintana poco después del primer boom turístico ${ }^{6}$ sobre la isla de Mallorca, iniciado en los años 1950-60, ya reflejara tan nítidamente la influencia de este sector.

\section{EVOLUCIÓN DEL TRABAJO DE QUINTANA, CON DATOS DE 2011}

Revisadas las conclusiones del trabajo de Quintana para 1970, veamos ahora cual sería la situación del sistema urbano de la isla si aplicamos su propio método de análisis a la realidad actual. Para ello, se cuenta con la Clasificación Nacional de Actividades Económicas de 2009 (CNAE09) como base de organización actual de los diferentes grupos de establecimientos que se han obtenido del Directorio Central de Empresas (DIRCE), el cual cuenta con el número de establecimientos que existen en Mallorca, a escala municipal, y provenientes de declaraciones o inscripciones activas para el 01 de enero de 2011. Esta clasificación será contrastada con los datos de la Matricula Industrial de Mallorca (Licencia Fiscal) de 1970, utilizada por Quintana.

A partir de la clasificación del DIRCE, para 2011 contamos con 22.560 establecimientos clasificados en 57 grupos. Esta primera aproximación nos muestra que actualmente existe un menor número de establecimientos y una mayor diversidad que la detectada por Quintana en su tesis. Sin embargo, hay que tener en cuenta que ya el propio autor adelantaba «es posible que la determinación de las funciones según las ramas de la Matrícula Industrial delimite categorías demasiado amplias para constituir efectivamente funciones diferentes.»(Quintana, 1979: 89). Para 2011, debe considerarse que se ha producido asimismo un aumento poblacional de 873.414 habitantes respecto a la población censada en 1970, lo que supone un aumento del 52,7\%.

Esta diferencia inicial de 9.708 establecimientos más en 1970 que en 2011 viene ocasionada, muy probablemente, al hecho de que la Licencia Fiscal ofrecía unos grupos más amplios de actividades y no permitía el mismo grado de detalle que el CNAE09, un ejemplo claro está citado por el propio Quintana (1979: 93):

«En la rama $1^{\mathrm{a}}$ de Servicios de la Matrícula Fiscal, incluye los establecimientos dedicados a las siguientes actividades: «Pupilaje de ganado, mataderos, operaciones de mediación y acopio; hostelería de hoteles, albergues, pensiones, fondas, casas de viajeros y huéspedes, paradores, posadas y mesones, hoteles-apartamentos, moteles; restaurantes, cafeterías, heladerías, y horchaterías; campamentos turísticos.»

6 Se entiende por boom turístico una etapa de fuerte crecimiento de este sector, a causa de una elevada llegada de turistas a la isla. El término «boom» se aplica a un acontecimiento que se desarrolla de forma rápida pero, que también acaba precipitadamente, debido a un cambio de tendencia. 
Así pues, como vemos en la rama $1^{\text {a }}$ de la Matrícula Fiscal, se incluye un grupo de establecimientos que engloba desde la ganadería a la hostelería, mientras que el CNAE09 clasifica los datos de forma mucho más detallada, sin quedar excluido, ni incluido, ningún tipo de establecimiento que no sea necesario.

Hechas estas aclaraciones, vamos a analizar en las Figuras 1 y 2, la comparación de los datos con los que trabajó Quintana para 1970 y con los que se ha trabajado para 2011.

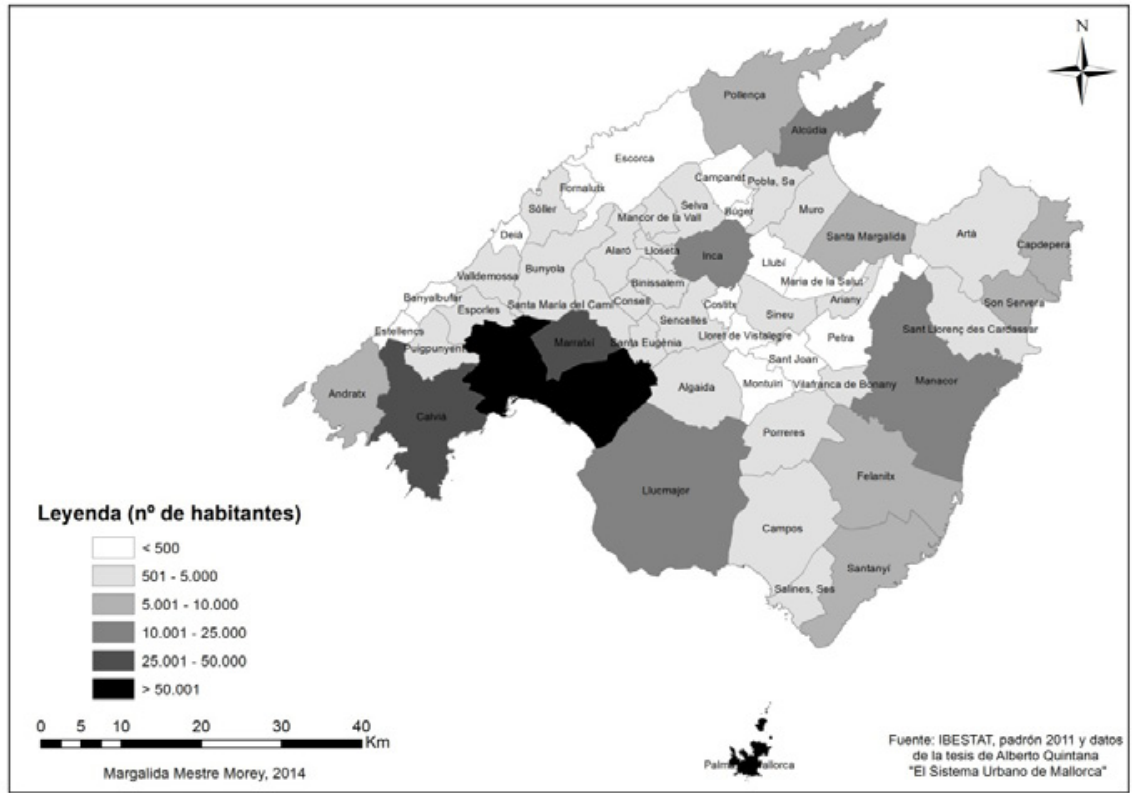

La figura 1 muestra tres aspectos clave:

a. Un aumento más significativo de la población en los municipios costeros, frente a los del interior.

b. La importancia de Palma y de sus municipios colindantes, influencia que se hace patente en la primera, e incluso la segunda, corona de municipios hacia el interior de la isla, remarcándose la importancia del eje Palma-Inca-Alcúdia.

c. El escaso crecimiento de los municipios de interior más alejados de la capital y de los municipios de la Serra de Tramuntana, a excepción de Sòller.

Cabe puntualizar que hay tres municipios con cifras negativas de población (Petra, Sant Joan y Estellencs). Aun así, la principal diferencia entre los dos años base es que aparece un municipio más en los datos de 2011, Ariany, el cual se independizó de Petra en 1982. Es en gran parte por este motivo que Petra tiene un saldo de población negativo de -1.048 habitantes. 
A parte de tener en cuenta que municipios han aumentado más o menos su cantidad de población, para analizar la integración del sistema urbano, es imprescindible conocer la variación porcentual que supone la cantidad de población de cada municipio, respecto al total de la isla, entre los dos años base.

Tabla 1

AUMENTO O DISMINUCIÓN EN LA DIFERENCIA PORCENTUAL DE POBLACIÓN ENTRE 1970 Y 2011

\begin{tabular}{cc}
\hline Municipios & $\%$ \\
\hline Calvià & $4,94 \%$ \\
\hline Marratxí & $2,94 \%$ \\
\hline Alcúdia & $1,33 \%$ \\
\hline Llucmajor & $1,27 \%$ \\
\hline Palma & $-4,48 \%$ \\
\hline
\end{tabular}

Fuente: elaboración propia a partir de datos del IBESTAT.

La tabla 1 muestra los municipios que han aumentado o disminuido $+/-1 \%$, viendo que únicamente la capital insular ha perdido peso porcentual de forma significativa, pasado del $50,9 \%$ en 1970, al 46,4\% en 2011. En gran medida, el peso que ha perdido Palma ha ido en favor de sus municipios colindantes, Calvià, Marratxí y Llucmajor, así como también de Alcúdia, municipio que contiene el segundo puerto más importante de la isla. Estos porcentajes reflejan una redistribución de la población sobre la isla, reequilibrando las diferencias entre la capital insular y el resto del territorio (Mestre, 2015).

Hechas estas constataciones, veamos ahora cuál es la distribución de las pérdidas de los casi 10.000 establecimientos desaparecidos entre 1970 y 2011 sobre el territorio.

La figura 2, muestra también tres aspectos clave:

a. El municipio que más establecimientos pierde, con una grandísima diferencia sobre los demás es Palma (-6.963).

b. Otros 43 municipios pierden establecimientos.

c. Solo 9 municipios han visto incrementar su número de establecimientos, se trata de los municipios de Alcúdia, Andratx, Calvià, Marratxí,Valldemossa, Fornalutx, Sant Llorenç des Cardassar, Santanyí y Ariany ${ }^{7}$. Como puede comprobarse se trata principalmente de municipios costeros y, por lo tanto, con intensa actividad turística, a excepción de Marratxí y Fornalutx.

Veamos a continuación cual es la distribución sobre la isla de los municipios que más han visto aumentar o disminuir su peso porcentual, permitiéndonos conocer si se han redistribuido en el mismo sentido que la población.

7 Ariany es una excepción, tiene saldo positivo debido a que es un municipio de nueva aparición, causando una mayor pérdida (ahora si más justificada) de establecimientos en Petra. 


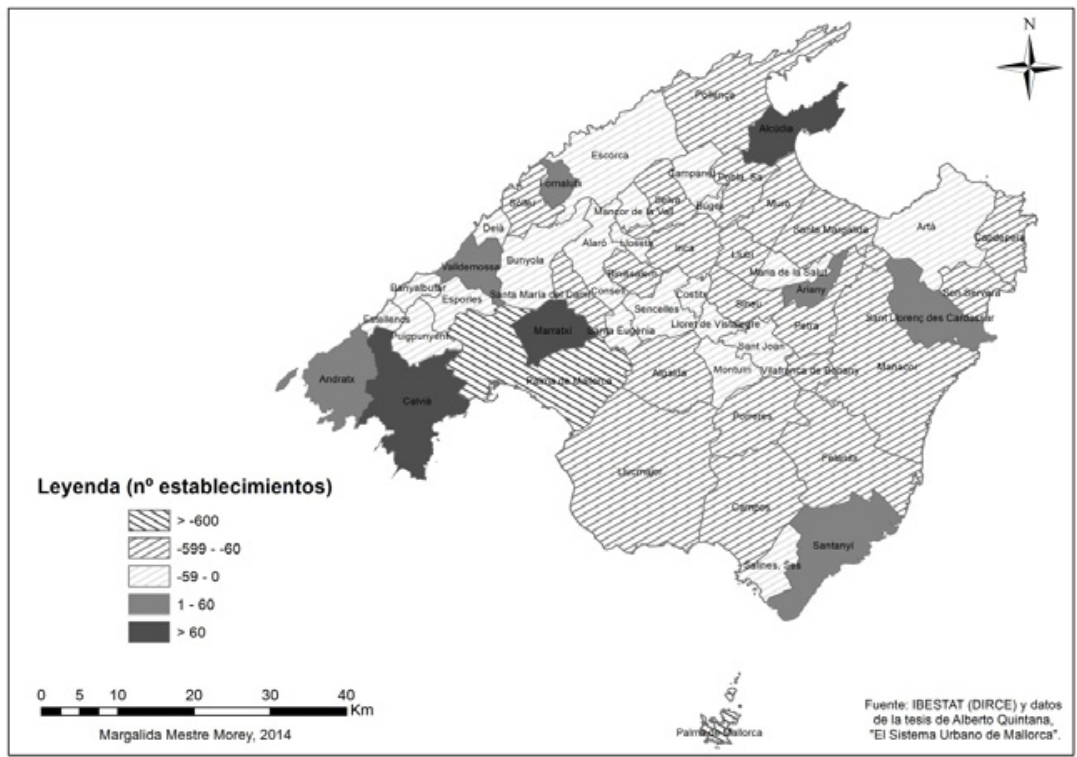

Tabla 2

AUMENTO O DISMINUCIÓN DE LA DIFERENCIA PORCENTUAL DE ESTABLECIMIENTOS ENTRE 1970 Y 2011

\begin{tabular}{cc}
\hline Municipios & $\%$ \\
\hline Calvià & $4,26 \%$ \\
\hline Alcúdia & $1,49 \%$ \\
Marratxí & $1,23 \%$ \\
\hline Palma & $-7,62 \%$ \\
\hline
\end{tabular}

Fuente: elaboración propia a partir de datos del IBESTAT.

Mediante la simple ordenación de municipios de mayor a menor, y reflejando en la Tabla 2 solo los que tienen un aumento o disminución $+/$ - el $1 \%$, al igual que en la tabla 1 , vemos que la mayor disminución se ha producido de nuevo en el municipio de Palma, mientras que los municipios que más han aumentado el número de establecimientos son dos de los tres sobre los que se extiende el continuo urbano de la capital (Mestre, 2013). Por otra parte, localizado en la bahía norte esta Alcúdia, municipio que al igual que ha ocurrido con la población (Tabla 1) la cantidad de establecimientos también ha aumentado de forma significativa.

Contrastando estos datos vemos que, pese a la pérdida de -9.708 establecimientos entre 1970 y 2011, existe una relación entre las variaciones relativas al peso de la población y de los establecimientos sobre la isla. Ello comporta la pérdida de peso de la capital y, como consecuencia, una redistribución de ambas variables sobre el territorio insular. 
Con la finalidad de seguir analizando esos primeros indicios de integración del sistema urbano vamos, a continuación, a llevar a cabo el análisis comparativo del resultado de las correlaciones entre los dos años base. Además de las correlaciones, rehaciendo el trabajo de Quintana, se actualizarán también, para 2011, las dos tipologías de establecimientos creadas con la finalidad de conocer la importancia comercial de los municipios mallorquines.

\section{ANÁLISIS DE LAS CORRELACIONES}

Reelaborado el trabajo de Alberto Quintana se tratará de determinar si las correlaciones con datos de 2011 denotan una relación diferente o en la misma dirección que los resultados de 1970. Para ello analizaremos, de la misma forma que hizo el autor, la diversidad y el número de establecimientos y, por otra parte, el número de establecimientos y la población.

Tabla 3

CORRELACIONES (R) DEL EQUIPAMIENTO COMERCIAL DE LOS MUNICIPIOS: COMPARACIÓN ENTRE 1970 Y 2011

\begin{tabular}{lcc} 
& \multicolumn{2}{c}{ Resultados } \\
\cline { 2 - 3 } & $\mathbf{1 9 7 0}$ & $\mathbf{2 0 1 1}$ \\
\hline $1^{\mathrm{a}} \cdot \mathrm{n}^{\mathrm{o}}$ de establecimientos y diversidad & $0,737^{8}$ & 0,363 \\
\hline $2^{\mathrm{a}} \cdot \mathrm{n}^{\mathrm{o}}$ de establecimientos y la población & 0,997 & 0,997 \\
\hline $3^{\mathrm{a}}$. Población y $\mathbf{n}^{\mathbf{o}}$ de establecimientos de los municipios turísticos & 0,725 & - \\
\hline $4^{\mathrm{a}}$. Población y $\mathbf{n}^{\mathbf{0}}$ de establecimientos de los municipios no turísticos & 0,951 & - \\
\hline
\end{tabular}

Fuente: Quintana (1979) y elaboración propia a partir de datos del IBESTAT.

Como ya sabemos, con la primera correlación se pretende saber si, a mayor número de establecimientos hay una mayor diversificación de los diferentes grupos clasificados por el CNAE09, mientras que con la segunda se indica si hay una relación donde a mayor número de establecimientos, más cantidad de población empadronada. En cuanto a la división entre municipios turísticos y no turísticos, en el año 1970 podía llevarse a cabo debido a que la isla estaba inmersa en el primer boom y no todos los municipios contaban con establecimientos turísticos ${ }^{9}$, pero para 2011, después del segundo y tercer boom, no existen razones objetivas que puedan excluir un municipio de la influencia del turismo (Artigues, 2007).

Para ajustar y poder llegar a una mejor aproximación de los datos, teniendo en cuenta que Quintana trabajó con 18 grupos de establecimientos y que mediante la clasificación del CNAE09 se han obtenido 57, se ha procedido a una reclasificación de las categorías recogidas en esta última fuente en 23 grupos (Anexo; Tabla 4).

A partir del reajuste de los datos iniciales, en cuanto a la primera correlación, el coeficiente resultante reafirma el resultado inicial de 1970, indicando que a mayor número de establecimientos no hay una mayor diversificación de los diferentes grupos. Ello confirma

8 Respecto a este dato, véase lo expresado en la nota 5.

9 Consultar Tabla 86 del Sistema urbano de Mallorca (Quintana, 1979). 
la fuerte especialización del comercio al por menor y del sector servicios de la que ya hablaba Quintana y también la fuerte urbanización del territorio, debido a la imposibilidad, de nuevo, de establecer una jerarquía «stricto sensu» entre el número de establecimientos y su diversidad.

Por otro lado, a partir de la segunda correlación se da, como hemos visto, el mismo resultado en 1970 y $2011(\mathrm{r}=0$,997). Que siga manteniéndose esta perfecta correlación refleja que actualmente sigue habiendo relación entre los municipios que tienen más población y más establecimientos. Este resultado podía intuirse después de encontrarnos en el apartado anterior con que ganan y pierden en la variación de peso porcentual casi los mismos municipios tanto en la cantidad de población, como de establecimientos. De esta forma, si en cuanto a la población ya se ha demostrado que ha habido una redistribución sobre el territorio (Mestre, 2015), un resultado tan ajustado entre las dos variables, nos muestra cómo, al menos a nivel municipal, se ha producido también una redistribución de los establecimientos. Pero, debido a que las características que definen la centralidad en los últimos 40 años han cambiado, para entender este resultado vamos a tomar como referencia la conclusión general a la que llegó Quintana al aplicar esta metodología: «la TLC solo puede ser aplicada en áreas con una población rural más o menos regularmente distribuida y donde no existan funciones especializadas como la del turismo, que alteren la centralidad de los lugares»(Quintana, 1979:89).

Como bien sabemos, el turismo es el motor que mueve la economía isleña y que influye en todas las dinámicas que rigen la isla. Por ello, cabe destacar que existen ciertos grupos de establecimientos aquí estudiados, como «Alojamientos turísticos» $\mathrm{y}$ «Alquiler de vehículos», que pueden considerarse turísticos, debido a que son los turistas los que realizan su consumo (Lanquar, 1991), y de los cuales ha de tenerse en cuenta que su localización en un determinado punto del territorio no viene motivado por la cantidad de población que allí reside, sino debido a una serie de atractivos y oportunidades que hacen que aquel lugar sea, por así decirlo, apetecible para los turistas (Riera, 1988).

Ese cambio en la lógica de la localización, desvirtúa el concepto de centralidad con el que trabaja la TLC y que, por tanto, como bien dijo Quintana, la hace en buena medida inservible. Pero, si se le incorpora a este concepto la lógica turística, se entendería por centralidad la ubicación de un establecimiento en función de la población o que la población se ubique en un punto del territorio debido a la presencia de un determinado tipo de establecimiento.

Debido a que actualmente la localización de establecimientos y de población en la isla se rige por esta última definición, la cual no rompe con la concepción intrínseca de centralidad, nos encontramos con que el coeficiente de correlación obtiene el mismo resultado.

Ahora bien, cabe matizar que si se variara la escala de análisis, el resultado de la correlación probablemente no sería tan perfecto. Si existieran datos suficientes para llevar a cabo el mismo análisis, pero con fracciones de territorio más pequeñas, es de suponer que la distribución de las dos variables sobre el territorio tendría fuertes descompensaciones.

Debido a que para el coeficiente de correlación la escala de medida de las variables es independiente, no estamos conociendo si la cantidad de establecimientos es suficiente para la población empadronada, sino más bien que existe una relación que vincula el volumen de población, con el número de establecimientos o viceversa.

Por otra parte, hay que tener en cuenta otros datos hasta ahora no reflejados y que podrían alterar estos resultados, como son la variabilidad de población fluctuante y de inscripción de 
establecimientos con los que cuenta la isla de Mallorca a lo largo del año. Para analizar este fenómeno podemos ver, en primer lugar, mediante el Índice de Presión Humana ${ }^{10}$ (IPH), el promedio mensual de población real que llega a haber sobre la isla, contrastándolo con los datos de población empadronada con los que estamos trabajando.

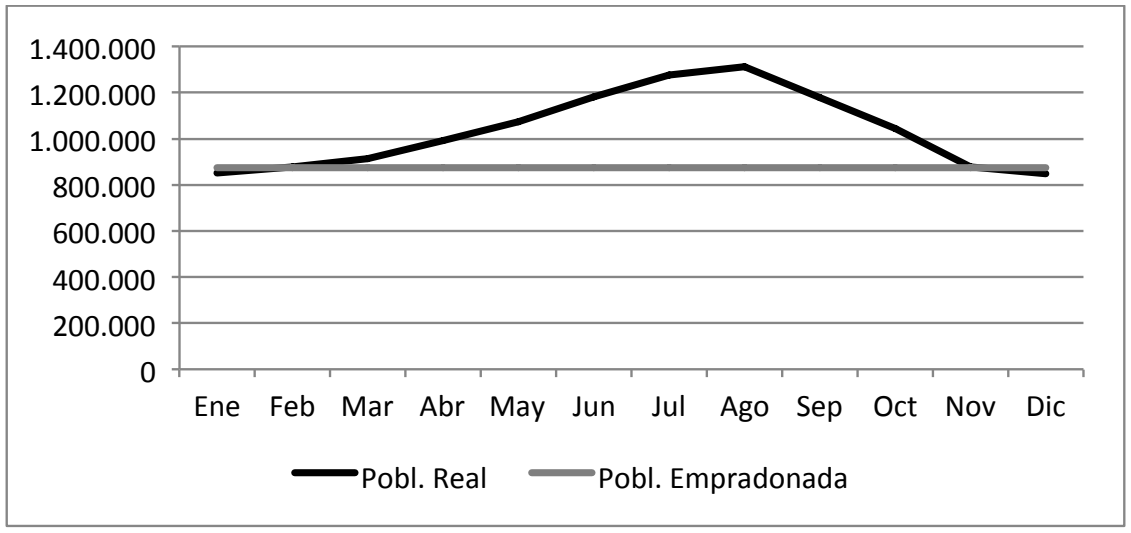

Fuente: elaboración propia a partir de datos del IBESTAT.

La Figura 3 muestra que en la isla existe un incremento medio sobre la población empadronada de más de 400.000 personas en agosto. Habiendo una variación tan amplia que en los meses de diciembre y enero se encuentra sobre la isla menos población de la que contabiliza el padrón.

En segundo lugar, y debido a que las consecuencias de la llegada estacional de turistas repercuten directamente sobre los demás aspectos de la isla, veamos que ocurre con la cantidad de establecimientos. Así como refleja la Figura 4, mediante la evolución de las altas y bajas en las cuentas de cotización de las empresas ${ }^{11}$, puede intuirse que la cantidad de establecimientos al por menor y servicios también fluctúa a lo largo del año. Alcanzando, al igual que ocurre con la población real, su máximo en los datos de temporada veraniega.

A partir de esta especificación, en la cantidad de población real y la variabilidad en el número de establecimientos, vemos como los resultados de las correlaciones representan una instantánea de un momento concreto, sin posibilidad de poder cuantificar la variación real consecuencia de la actividad turística.

10 A partir de las llegadas y salidas diarias de pasajeros del aeropuerto, del puerto, estimaciones de población actual y proyecciones de población a corto plazo, el IBESTAT construye el IPH, que permite conocer la cantidad de población real diaria que hay sobre la isla.

11 Estos datos están condicionados por una serie de inconvenientes, como por ejemplo, que las empresas tienen la obligación de inscribir únicamente un mínimo de una cuenta de cotización por provincia en las que desarrollen su actividad. Pudiendo, diversas filiales de la misma provincia, compartir dicha cuenta de cotización. Es por ello que estos datos solo pretenden servir de ejemplo extrapolable a la estacionalidad que también existe para el número de establecimientos al por menor y servicios. 
Figura 4

EVOLUCIÓN DEL PORCENTAJE DE EMPRESAS QUE SE HAN DADO

DE ALTA EN LA SEGURIDAD SOCIAL EN CADA TRIMESTRE DE 2011

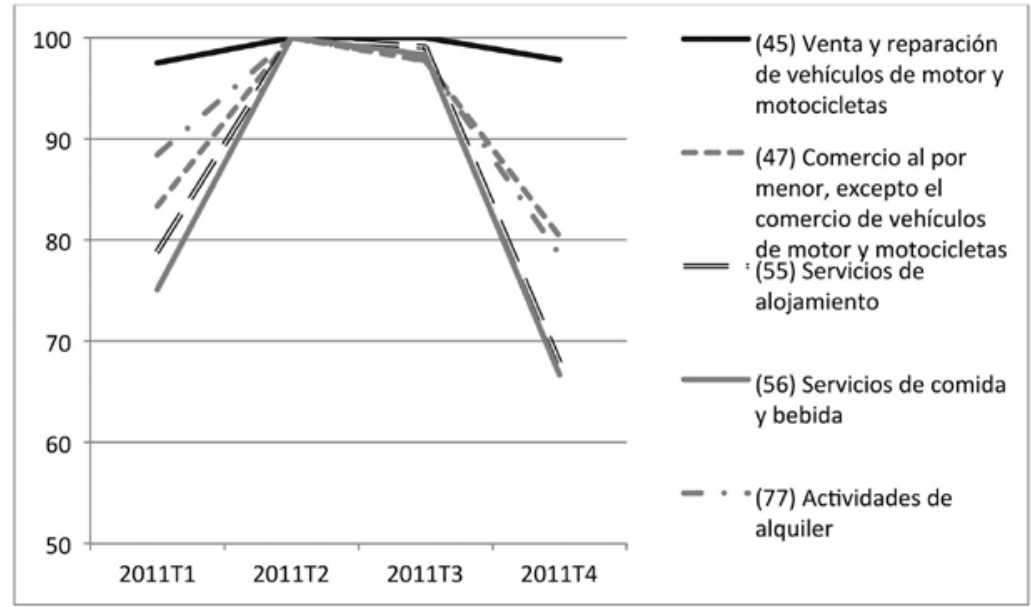

Fuente: elaboración propia a partir de datos del IBESTAT.

\section{TIPOLOGÍAS DEL EQUIPAMIENTO COMERCIAL DE LOS MUNICIPIOS}

Después de analizar los resultados de las correlaciones, Quintana construyó, con la intención de conocer cuál es la importancia absoluta y relativa de los establecimientos comerciales de cada municipio, dos tipologías que establecen diferentes rangos clasificatorios y que permitirán conocer, a escala municipal, más datos sobre la relación entre el número de establecimientos y la población, evaluando también el impacto de los establecimientos turísticos.

La primera tipología se construye a partir de datos relativos. El autor trabajó con porcentajes de número de población y de número de establecimientos de cada municipio, calculando qué peso tienen sobre el total las dos variables. Fue a partir de estos resultados que calculó la variación existente entre ambos, para saber qué municipios tienen un porcentaje sensiblemente igual -equilibrados- y cuales están desequilibrados, ya sea hacia el sobreequipamiento o el subequipamiento. Tal y como ya reconocía el propio autor «ahora bien, es claro que en una variación del cuatro o cinco por ciento, pongamos por caso, no representa lo mismo si la base de partida es una o es otra» (Quintana, 1979: 91), debido a esto se llevó a cabo una ponderación de la desviación entre las variables, calculando el porcentaje que la variación supone sobre la variable de base ${ }^{12}$.

Debido a que el resultado de la ponderación, de aquí en adelante llamado grado de variación ponderado (GVP), indica sólo la intensidad del desequilibrio sufrido por cada muni-

12 Quintana en su tesis expone un ejemplo representativo de dos municipios donde «en efecto, la variación en la participación sobre los establecimientos y sobre la población es, en ambos municipios, sensiblemente parecida. Pero es evidente que un 3 por ciento de diferencia no representa lo mismo si se parte de un 1 por ciento que si se parte de un 50 por ciento» (Quintana, 1979: 91). 
Figura 5

PRIMERA TIPOLOGÍA DE EQUIPAMIENTO COMERCIAL MUNICIPAL, 1970

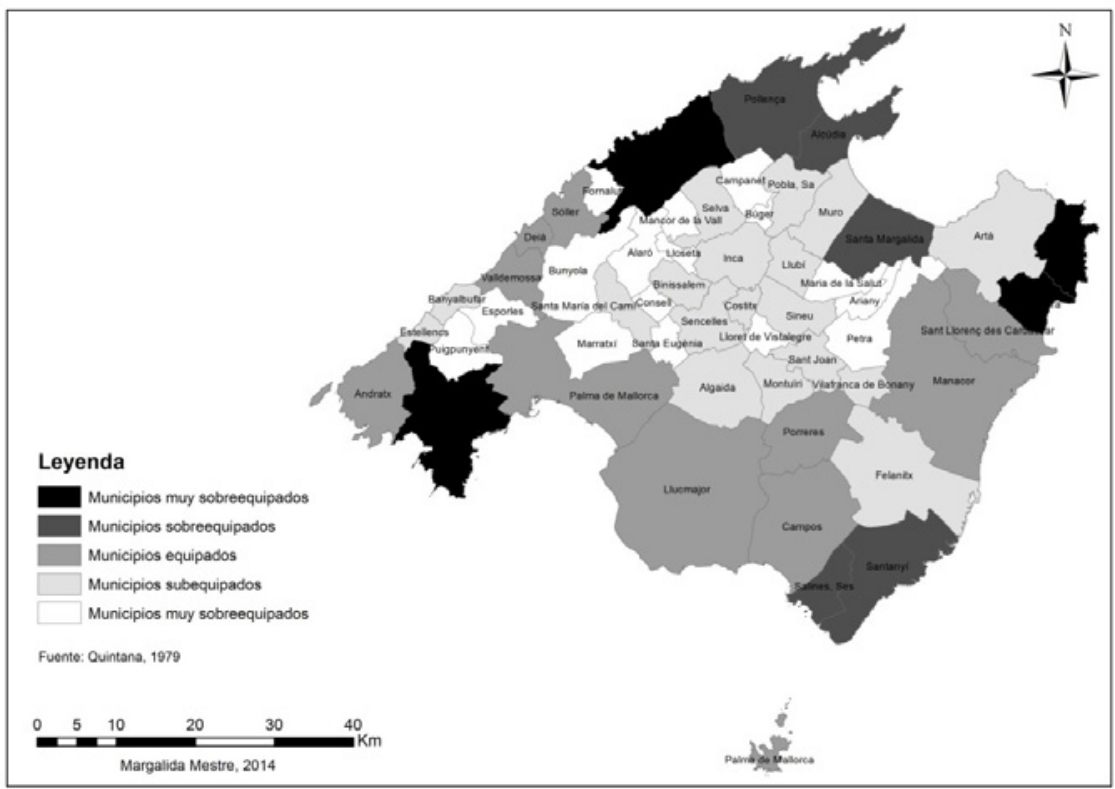

Figura 6

PRIMERA TIPOLOGIA DE EQUIPAMENTO COMERCIAL MUNICIPAL, 2011

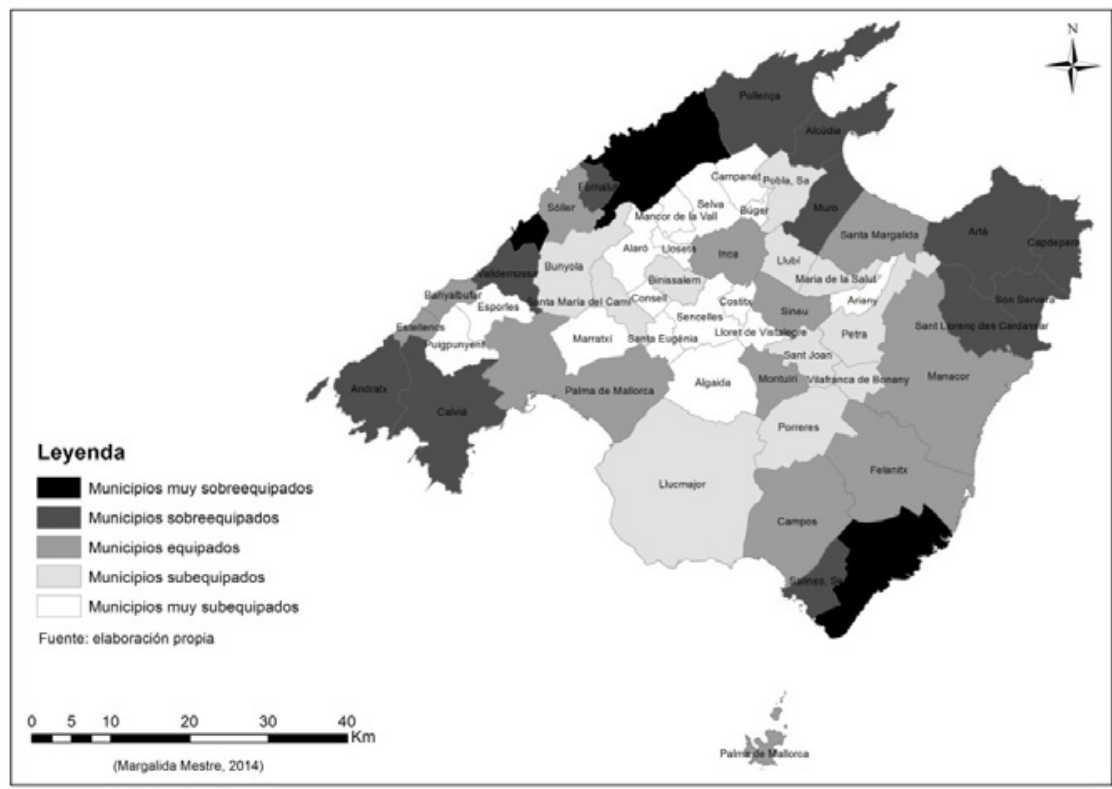


cipio, el autor introdujo una nueva variable, el número de establecimientos existentes en cada municipio por cada 1000 habitantes, con la intención de conocer si se encontraba con municipios sobreequipados, equipados o subequipados. Finalmente, la metodología para la clasificación de los municipios ha consistido en usar el método de la desviación estándar para las dos variables estudiadas.

El interés de esta clasificación yace en que permite conocer si la cantidad de establecimientos de cada municipio da respuesta a la cantidad de habitantes empadronados. Aunque, como explicó Quintana (1979: 93), el método utilizado tiene una serie de deficiencias:

- Al emplear dos variables que en buena parte son similares, el resultado sólo informa del mayor o menor equipamiento en relación con la población residente.

- Por otra parte, de alguna forma se podrían ver favorecidos por esta clasificación los municipios costeros, que soportan más directamente el elevado número de establecimientos relacionados con el turismo de sol y playa.

Comparando los mapas de los dos años base (Figuras 5 y 6), los principales cambios que ha experimentado el sistema urbano mallorquín, han consistido en:

a. Un reforzamiento el equipamiento comercial costero del norte, del levante y de la sierra de Tramuntana.

b. Una disminución de la importancia comercial de los municipios de Llucmajor, Calvià y Algaida, debido a la redistribución de la población y los establecimientos.

c. Un equilibrio comercial de los municipios de interior de la isla, como Inca, Sineu y Montuïri. Mientras que por el contrario Costitx, Senselles y Selva, entre otros, han perdido importancia.

Aunque, con todo ello, hay que tener en cuenta que también influye la escasa dimensión de la isla, permitiendo desplazamientos a cualquier punto de forma rápida, dejando de ser la centralidad una condición vital que relacione la localización con el consumo.

Con la finalidad de corregir las deficiencias de la primera tipología vamos a trabajar con datos absolutos y, de la misma manera que lo hizo Quintana ${ }^{13}$, teniendo únicamente en cuenta los establecimientos considerados como no turísticos ${ }^{14}$. Con ello se persigue trabajar con el número de establecimientos que más se ajuste a los que sirven a la población empadronada.

13 Cabe tener en cuenta que Quintana para excluir aquellos establecimientos al por menor y servicios turísticos, tuvo que excluir también otros grupos de establecimientos considerados como no turísticos, esto es debido a la escasa diversidad de las ramas de la Matricula Fiscal de 1970, aunque el autor lo justifica debido a que «creemos que en el conjunto de estos establecimientos [ver Quintana, 1979: 93] el peso turístico es tan aplastante que queda ampliamente justificada su exclusión para nuestros objetivos».

14 En 2011, y según el CNAE09, se consideran turísticos los siguientes tipos de establecimientos: «5510 - Hoteles y alojamientos similares; 5520 - Alojamientos turísticos y otros alojamientos de corta estancia; 5530 - Cámpings y aparcamiento para caravanas; 5590 - Otros alojamientos», además, también se han clasificado como turísticos los siguientes, debido a que su elevada frecuencia sobre el territorio es consecuencia de la actividad turística: «7711 - Alquiler de automóviles y vehículos de motor ligeros; 7734 - Alquiler de medios de navegación; 5610 - Restaurantes y lugares de comidas; 5621 - Provisión de comidas preparadas para eventos; 5629 - Otros servicios de comidas; 5630 - Establecimientos de bebidas». 
Figura 7

SEGUNDA TIPOLOGÍA DE EQUIPAMIENTO COMERCIAL MUNICIPAL, 1970

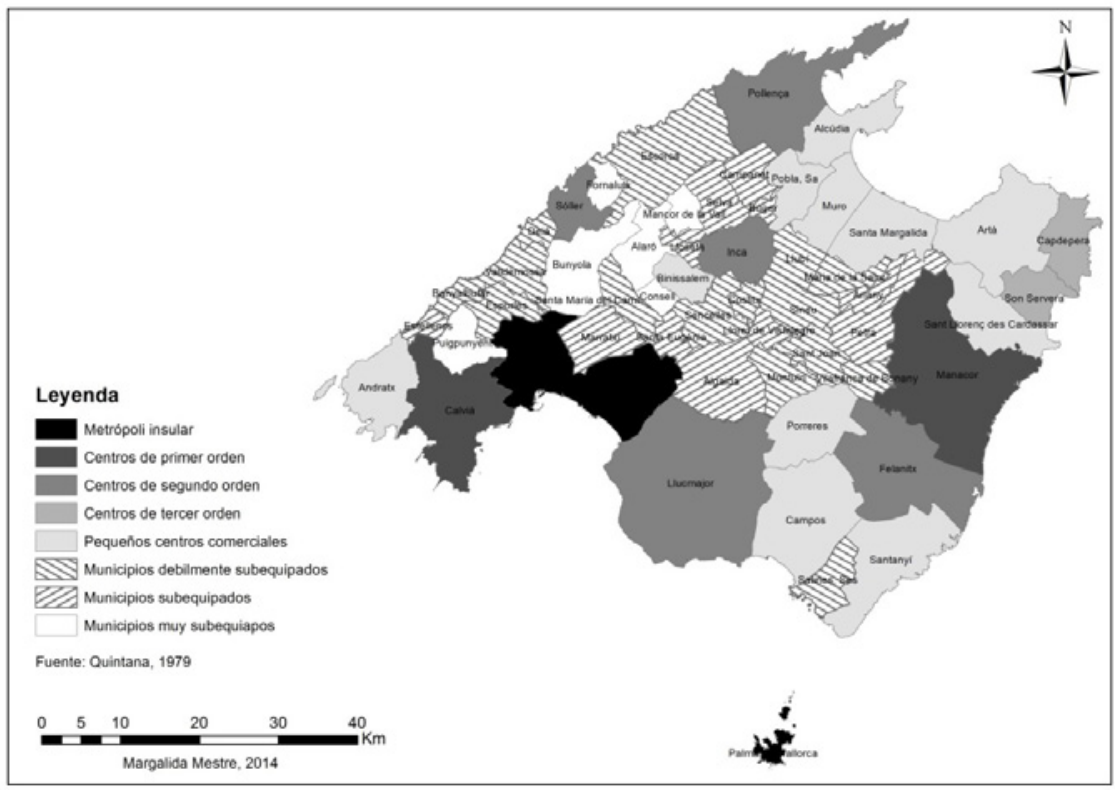

Figura 8

SEGUNDA TIPOLOGIA DE EQUIPAMIENTO COMERCIAL MUNICIPAL, 2011

\section{Leyenda}
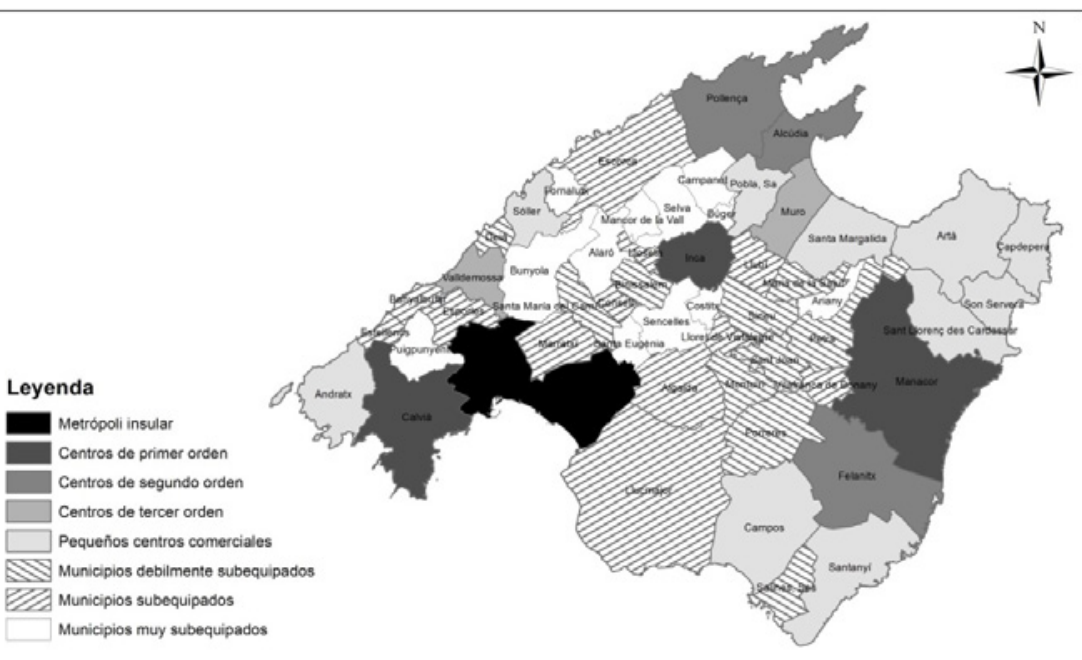

Municipios subequipados

Municipios muy subequipados

Fuente: elaboración propia
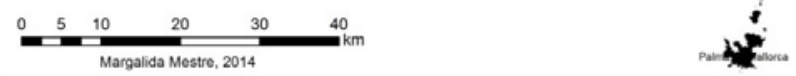
Para elaborar la segunda tipología se han tenido en cuenta los datos referidos al GVP, igual que en la tipología anterior, pero a diferencia de esta, se ha tenido en cuenta la cantidad absoluta de establecimientos comerciales (excepto los establecimientos turísticos) para poder conocer si el grado de desequilibrio que presentan los municipios va hacia al sobreequipamiento o al subequipamiento. Con ello se corrigen las deficiencias de la tipología anterior, evitando trabajar con datos relativos de establecimientos en las dos variables.

Aun así, a pesar de aplicar la misma tipología que Quintana, la clasificación de los municipios para 2011 tiene diferentes rangos cuantitativos debido a la necesidad de adaptar las nuevas cantidades, tanto de población, como de establecimientos. Pero, pese a esas diferencias se a utilizando, de la misma forma que en la primera tipología, el método de la desviación estándar para la clasificación de los municipios.

La importancia de esta segunda tipología consiste en que permite mitigar la influencia del turismo en el sistema comercial, pudiendo así tener un reflejo de cuál es la estructura comercial del sistema urbano para los residentes. Comparando las dos figuras puede observarse como:

a. En los últimos 40 años se ha reforzado la importancia de Inca. El hecho que este municipio, juntamente con Palma-Calvià y Manacor, forme parte de los centros de primer orden, hace resurgir, pese a la indiscutible influencia turística, la estructura tripoidal del sistema urbano preturístico (Rullan, 2002: 321-6).

b. Los municipios costeros mantienen mayoritariamente su importancia comercial. Destacándose como casos opuestos Alcúdia y Llucmajor, los cuales, como enclaves de diferente época de explosión turística, parecen responder a causas contrarias de redistribución, tanto comercial como poblacional. Los municipios costeros, por su importancia turística, han sido concentradores de población y establecimientos des de los años 1960.

c. Hay un mayor número de municipios de interior subequipados en 2011 que en 1970, tal vez debido a los polos de atracción que suponen las cabeceras de Palma-Calvià, Inca y Manacor-Felanitx.

En conclusión, la diferencia principal entre la primera y la segunda tipología recae en la influencia del sector turístico, donde la segunda tipología respondería, en mayor medida, a los establecimientos más frecuentados por la población residente. Como nos muestra la Figura 8 sigue aún presente la estructura urbana en modelo de triángulo o en forma de «Y» del sistema urbano preturístico, que caracterizaba a los primeros estudios del sistema comercial insular (Fontana, 1963). Aunque este modelo ya está altamente influenciado por el turismo, influencia que ya se iniciaba en el estudio de las áreas comerciales de 1988 (Rullan, 2002) y que ahora ha aumentado, no únicamente en Calvià, sino también en la importancia de, sobretodo, Felanitx, Pollença y Alcúdia.

\section{CONCLUSIÓN}

A partir del estudio del sistema urbano de Mallorca en base a la evolución de los establecimientos comerciales al por menor y los servicios en 1970 y en 2011, se ha llegado a la afirmación que, a lo largo de estos 40 años el sistema urbano mallorquín ha modificado de manera notable su jerarquía. Hemos tratado de demostrar este hecho a través del análisis de las correlaciones, 
las cuales, además de mostrarnos un sistema comercial más especializado que en 1970, nos han permitido constatar la redistribución, tanto de la población, como de los establecimientos.

Además, cabe señalar que dicha redistribución se rige por criterios de centralidad, aunque, probablemente se trate de una centralidad diferente a la que define la TLC, debido a que es ejercida también por los propios establecimientos turísticos, los cuales se localizan en el territorio debido a ciertos atractivos y oportunidades.

Por otra parte, mediante las tipologías clasificatorias construidas por el propio Quintana, se ha podido ver como la importancia de los diferentes municipios dentro del sistema comercial de la isla varía en función de si se trabaja con el total de establecimientos, o únicamente con aquellos no turísticos. En ausencia de los cuales resurge, aunque ya de forma difuminada debido a la huella imborrable del turismo, el sistema urbano preturístico, estructurado a partir de los municipios de Inca, Palma y Manacor.

También debe señalarse el papel de Palma dentro del sistema urbano. Aunque actualmente tenga dentro de sus límites municipales el 46,6\% de la población y el 46,1\% de los establecimientos de toda la isla, no se encuentran allí ubicados, como ya demostraba Quintana, todos los grupos clasificados por el CNAE-09. De esta forma, partiendo de la redistribución del peso porcentual ya mencionada y del hecho de que el $100 \%$ de diversidad no se encuentre en la capital insular, hace perder importancia a la característica que hasta ahora más bien definía el conjunto del sistema urbano isleño, la acusada jerarquía.

Para finalizar, volviendo a la tesis doctoral de 1979, y partiendo, de entre otros resultados, del hecho que en la primera tipología Palma constase solamente como municipio equipado, Quintana expuso la siguiente conclusión «¿No obedecería esa aparente carencia de Palma a una efectiva falta de centralidad, es decir, a no constituir la capital regional, sino sólo un núcleo intermedio?» (Quintana, 1979: 89). Esta reflexión innovadora en los años 1970, actualmente puede considerarse plenamente incorporada en el análisis geográfico. Debido a la revolución de la tecnología en los últimos 40 años, donde el transporte y las comunicaciones han roto, en parte, con la importancia de la localización comercial, encontrándonos actualmente con una distribución de la diversidad de establecimientos sobre el territorio que no responde a los principios de centralidad que marcaba la entonces novedosa Teoría de los Lugares Centrales. Esto implica que la Mallorca actual no necesita de una ciudad cabecera que cumpla con el $100 \%$ de la diversidad de establecimientos, sino que es necesaria la concepción de integración de todo el sistema urbano para convertirse en un único centro de primer orden, incluso a escala europea, a partir de su especialización en servicios turísticos.

\section{BIBLIOGRAFÍA}

ARTIGUES, A.A. (2007): «Funcionalización turística y proceso de urbanización en la isla de Mallorca» en Introducción a la Geografía Urbana de las Illes Balears. Guía de campo. Palma. Universitat de les Illes Balears y Asociación de Geógrafos de España.

ARTIGUES, A.A; BLÁZQUEZ, M; GUAL, J; ET AL. (2008): Albert Quintana: el seu temps i la seva obra. Quaderns de territori, Vol. 01 . Govern de les Illes Balears.

BARCELO, B. (1968): Les illes Balears. Barcelona. Tàber.

BARCELÓ, B. (1970): Evolución reciente y estructura actual de la población en las islas Baleares. Tesis Doctoral. Madrid. CSIC. 
BERRY, B. (1971): Geografía de los centros de mercado y distribución al por menor. Barcelona. Edit. Vicens-Vives.

BISSON, J. (1977): La terre et l'homme aux iles Baleares. Aix-en-Provence. EDISUD

CAPEL, H. (2003): «Una mirada histórica sobre los estudios de redes de ciudades y sistemas urbanos» en Geotropico, 30-65, núm. 1 (1).

www.elcolegiodehidalgo.edu.mx/principal/archivos/inegi/metodologia/2.2/Capel, $\% 20$ 2003(2).pdf_(02/06/2014)

CAPEL, H. (2008): «Una mirada histórica sobre los estudios de redes de ciudades y sistemas urbanos. La tesis doctoral de Alberto Quintana en la geografía española en los años 1970» en Albert Quintana, el seu temps i la seva obra. Quaderns del territori, Vol. 01, 431-464.

CHRISTALLER, W. (1933): Die zentralen Orte in Süddeutschland. Jena. Gustav Fischer

COCIN (1964): «Áreas y subáreas comerciales de Mallorca e Ibiza en 1962» en Memoria Comercial y de trabajo. Madrid. Cámara Oficial de Comercio, Industria y Navegación, 43-55.

DEFFONTAINES, P (1967): «Islas Baleares» en Geografía de España y Portugal. Vol. IV. Barcelona. Montaner y Simón, 173-227.

ESTÉBANEZ, J. (1974): Cuenca. Estudio Geográfico. Madrid. Editado por el Instituto de Geografía Aplicada, Patronato de 'Alonso de Herrera' CSIC.

FERRER, M (1974): Población y propiedad en la cordillera septentrional de Mallorca. (Evolución histórica). Palma. Diputación Provincial de Baleares (2 volumenes).

FLORIT, F (1983): Les transformacions del paisatge rural i de la propietat de la terra a la comarca del Pla de Mallorca (XIX-XX). Tesis doctoral. UIB.

FONTANA, J.M. (Ed.) (1963): Atlas comercial de España. Madrid. Consejo Superior de Camaras de Comercio, Industria y Navegación de España.

LANQUAR, R. (1991): La economia del turismo. Barcelona. Oikos-tau.

LOPEZ, L. (1987): «Los estudios sobre lugares centrales en España y Portugal» en Anales de Geografía de la Universidad Complutense. Vol. 7, 449-459.

LÖSCH, A. (1944): Die räumliche Ordnung der Wirtschaft. Jena. Gustav Fischer.

MESTRE, M. (2013): «La delimitació de l'àrea urbana de Palma de Mallorca. Una reconsideració a partir de l'aplicació del criteri NUREC» en Treballs de la Societat Catalana de Geografia, n $^{\circ} 76,103-126$.

MESTRE, M. (2015): «Ciutat i territorio a Mallorca. Una revisió de la macrocefalia mallorquina» en Documents d'Anàlisi Geogràfica, vol. 61/2, 351-368

QUINTANA, A. (1974): «Espacio y sociedad en Mallorca» en Albert Quintana, el seu temps i la seva obra. (Artigues, A., et al, editors). Quaderns de territori, Vol.1, 185-195.

QUINTANA, A. (1979): El sistema urbano de Mallorca. Palma. Editorial Moll.

RIERA, P. (1988): Les àrees funcionals a Catalunya. Tesis doctoral. UAB

ROSSELLÓ, V. (1964): «El regadío en la isla de Mallorca» en aportación española al XX Congreso Internacional de Geografía. Madrid. CSIC, 235-254.

RULLAN, 0. (1979): «La previsió de sòl a Mallorca: una variable condicionada pel turisme i la segona residencia» en Treballs de Geografia, Departament de ciències de la terra, UIB. $\mathrm{n}^{\mathrm{o}} 42,119-128$

RULLAN, O. (2002): La construcció territorial de Mallorca. Palma, Editorial Moll.

SALVÀ, P.A. (1978): Aproximación al conocimiento de la transformación del espacio rural en la serra de Tramuntana de la isla de Mallorca. Tesis doctoral. UB 


\section{ANEXO}

Tabla 4

POBLACIÓN, NÚMERO I GRUPOS DE ESTABLECIMIENTOS DE LOS MUNICIPIOS MALLORQUINES, 2011

\begin{tabular}{|c|c|c|c|c|c|c|c|c|c|}
\hline 兽 & 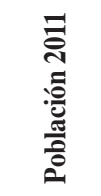 & 总 & 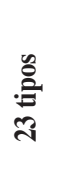 & 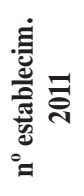 & 总 & 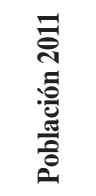 & 总 & 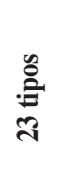 & 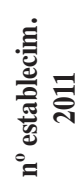 \\
\hline Palma & 405318 & 54 & 21 & 10469 & Ses Salines & 5272 & 29 & 15 & 162 \\
\hline Calvià & 52451 & 47 & 19 & 1897 & Esporles & 4956 & 25 & 14 & 74 \\
\hline Manacor & 40873 & 47 & 18 & 1125 & Consell & 3789 & 23 & 14 & 50 \\
\hline Llucmajor & 36959 & 44 & 18 & 690 & Selva & 3697 & 18 & 14 & 57 \\
\hline Marratxí & 34385 & 46 & 17 & 472 & Sineu & 3687 & 25 & 13 & 88 \\
\hline Inca & 29966 & 42 & 18 & 733 & Sencelles & 3128 & 17 & 11 & 36 \\
\hline Alcúdia & 19243 & 44 & 18 & 617 & $\begin{array}{l}\text { Villafranca de } \\
\text { Bonany }\end{array}$ & 2958 & 19 & 12 & 52 \\
\hline Felanitx & 18482 & 45 & 19 & 484 & Petra & 2919 & 26 & 15 & 58 \\
\hline Pollença & 16114 & 42 & 18 & 614 & Montuïri & 2872 & 24 & 14 & 72 \\
\hline Sóller & 14148 & 38 & 17 & 381 & Campanet & 2612 & 19 & 12 & 33 \\
\hline Santanyí & 13095 & 38 & 18 & 508 & Llubí & 2299 & 19 & 13 & 43 \\
\hline Sa Pobla & 12881 & 37 & 17 & 224 & $\begin{array}{l}\text { Maria de la } \\
\text { Salut }\end{array}$ & 2204 & 20 & 14 & 39 \\
\hline Son Servera & 12195 & 39 & 18 & 367 & Valldemossa & 2007 & 18 & 15 & 69 \\
\hline Andratx & 11919 & 40 & 18 & 398 & Sant Joan & 1985 & 17 & 12 & 40 \\
\hline Capdepera & 11858 & 41 & 17 & 437 & Puigpunyent & 1970 & 11 & 11 & 28 \\
\hline $\begin{array}{c}\text { Santa } \\
\text { Margalida }\end{array}$ & 11718 & 36 & 17 & 322 & Santa Eugènia & 1640 & 11 & 10 & 23 \\
\hline Campos & 9862 & 39 & 18 & 269 & $\begin{array}{l}\text { Mancor de la } \\
\text { Vall }\end{array}$ & 1324 & 9 & 8 & 18 \\
\hline $\begin{array}{c}\text { Sant } \\
\text { Llorenç des } \\
\text { Cardassar }\end{array}$ & 8963 & 35 & 17 & 310 & $\begin{array}{l}\text { Lloret de } \\
\text { Vistalegre }\end{array}$ & 1290 & 10 & 6 & 18 \\
\hline Binissalem & 7601 & 36 & 18 & 146 & Costitx & 1133 & 11 & 10 & 14 \\
\hline Artà & 7553 & 37 & 17 & 228 & Búger & 1047 & 10 & 9 & 18 \\
\hline Muro & 6970 & 35 & 18 & 248 & Ariany & 883 & 9 & 7 & 10 \\
\hline $\begin{array}{l}\text { Santa Maria } \\
\text { del Camí }\end{array}$ & 6270 & 33 & 16 & 112 & Deià & 752 & 12 & 10 & 35 \\
\hline Bunyola & 6232 & 28 & 16 & 77 & Fornalutx & 698 & 9 & 9 & 23 \\
\hline Lloseta & 5703 & 23 & 12 & 73 & Banyalbufar & 592 & 10 & 8 & 16 \\
\hline Porreres & 5544 & 31 & 17 & 98 & Estellencs & 402 & 6 & 6 & 11 \\
\hline Alaró & 5438 & 27 & 16 & 77 & Escorca & 284 & 4 & 4 & 16 \\
\hline Algaida & 5273 & 25 & 14 & 81 & Total & 873414 & & & 22560 \\
\hline
\end{tabular}

Fuente: elaboración propia a partir de datos del IBESTAT. 
